\title{
On nonlocal models of Kulish-Sklyanin type and generalized Fourier transforms
}

\author{
V. S. Gerdjikov
}

\begin{abstract}
A special class of multicomponent NLS equations, generalizing the vector NLS and related to the BD.I-type symmetric are shown to be integrable through the inverse scattering method (ISM). The corresponding fundamental analytic solutions are constructing thus reducing the inverse scattering problem to a RiemannHilbert problem. We introduce the minimal sets of scattering data $\mathfrak{T}$ which determines uniquely the scattering matrix and the potential $Q$ of the Lax operator. The elements of $\mathfrak{T}$ can be viewed as the expansion coefficients of $Q$ over the 'squared solutions' that are natural generalizations of the standard exponentials. Thus we demonstrate that the mapping $\mathfrak{T} \rightarrow Q$ is a generalized Fourier transform. Special attention is paid to two special representatives of this MNLS with three-component and five components which describe spinor ( $F=1$ and $F=2$, respectively) BoseEinstein condensates.
\end{abstract}

\section{Introduction}

The integrable multicomponent NLS (MNLS) equations are naturally related to the symmetric spaces [28, 9]. Formally the corresponding MNLS can be written as:

$$
i \frac{\partial \mathbf{u}}{\partial t}+\frac{1}{2} \frac{\partial^{2} \mathbf{u}}{\partial x^{2}}+\mathbf{u u}^{\dagger} \mathbf{u}=0
$$

see [11, 15]. Here $\mathbf{u}$ may be generic $k \times n$ rectangular matrix. It is well known that these MNLS are related to the A.III class of symmetric spaces in Cartan classifica-

V. S. Gerdjikov

Institute of Nuclear Research and Nuclear Energy, Bulgarian Academy of Sciences,

72 Tsarigradsko chausee, Sofia 1784, Bulgaria, and

Institute of Mathematics and Informatics, Bulgarian Academy of Sciences,

Acad. Georgi Bonchev Str., Block 8, 1113 Sofia, Bulgaria

e-mail: gerjikoveinrne.bas.bg 
tion. Of course, one should consider also the numerous MNLS that can be obtained from (1) by applying Mikhailov reductions [29], see [15, 13, 14, 16, 17, 19].

Some of these MNLS have applications to physics. Most of them are related to the vector NLS, i.e. $k=1$ and $\mathbf{u}$ is an $n$-component vector; for $n=2$ this is the famous Manakov model [28], see also [5, 8].

Another very interesting class of MNLS has been discovered by Kulish and Sklyanin [27]. The simplest nontrivial Kulish-Sklyanin (KS) model is a 3-component one

$$
\begin{aligned}
& i \partial_{t} \Phi_{1}+\partial_{x}^{2} \Phi_{1}+2\left(\left|\Phi_{1}\right|^{2}+2\left|\Phi_{0}\right|^{2}\right) \Phi_{1}+2 \Phi_{-1}^{*} \Phi_{0}^{2}=0, \\
& i \partial_{t} \Phi_{0}+\partial_{x}^{2} \Phi_{0}+2\left(\left|\Phi_{-1}\right|^{2}+\left|\Phi_{0}\right|^{2}+\left|\Phi_{1}\right|^{2}\right) \Phi_{0}+2 \Phi_{0}^{*} \Phi_{1} \Phi_{-1}=0, \\
& i \partial_{t} \Phi_{-1}+\partial_{x}^{2} \Phi_{-1}+2\left(\left|\Phi_{-1}\right|^{2}+2\left|\Phi_{0}\right|^{2}\right) \Phi_{-1}+2 \Phi_{1}^{*} \Phi_{0}^{2}=0 .
\end{aligned}
$$

Its integrability, both in classical and quantum sense, was demonstrated in [27], see also [17, 18, 26].

The next member in this class is a 5-component one:

$$
\begin{aligned}
& i \partial_{t} \Phi_{ \pm 2}+\partial_{x x} \Phi_{ \pm 2}=-2 \varepsilon\left(\Phi, \Phi^{*}\right)(x, t) \Phi_{ \pm 2}+\varepsilon \Theta(x, t) \Phi_{\mp 2}^{*}, \\
& i \partial_{t} \Phi_{ \pm 1}+\partial_{x x} \Phi_{ \pm 1}=-2 \varepsilon\left(\Phi, \Phi^{*}\right)(x, t) \Phi_{ \pm 1}-\varepsilon \Theta(x, t) \Phi_{\mp 1}^{*}, \\
& i \partial_{t} \Phi_{0}+\partial_{x x} \Phi_{0}=-2 \varepsilon\left(\Phi, \Phi^{*}\right)(x, t) \Phi_{0}+\varepsilon \Theta(x, t) \Phi_{0}^{*}
\end{aligned}
$$

where $\varepsilon= \pm 1$ and

$$
\begin{aligned}
\left(\Phi, \Phi^{*}\right)(x, t) & =\sum_{\alpha=-2}^{2} \Phi_{\alpha} \Phi_{\alpha}^{*}, \\
\Theta(x, t) & =\left(\Phi, s_{0} \Phi\right)=2 \Phi_{2} \Phi_{-2}-2 \Phi_{1} \Phi_{-1}+\Phi_{0}^{2} .
\end{aligned}
$$

Both KS models find important physical applications in describing spin-1 and spin-2 Bose-Einstein condensates (BEC). Indeed, BEC of alkali atoms in the $F=1$ hyperfine state, elongated in $x$ direction and confined in the transverse directions $y, z$ by purely optical means are described by a 3 -component normalized spinor wave vector $\Phi(x, t)=\left(\Phi_{1}, \Phi_{0}, \Phi_{-1}\right)^{T}(x, t)$ satisfying the equation (2), see [30, 36, 37, 8 , 25]:

The assembly of atoms in the hyperfine state of spin $F$ can be described by a normalized spinor wave vector with $2 F+1$ components

$$
\left.\Phi(x, t)=\left(\Phi_{F}(x, t), \Phi_{F-1}(x, t), \ldots, \Phi_{-F}(x, t)\right)^{T}\right)^{T},
$$

whose components are labeled by the values of $m_{F}=F, \ldots, 1,0,-1, \ldots,-F$. So the spinor BEC with $F=2$ (taken for rather specific choices of the scattering lengths) in dimensionless coordinates takes the form (3) [37, 17]. For those who are interested in the physics of spinor BEC we provide some more relevant references [39, 34, 30, 36, 33, 38].

In the last decade a new trend was started in nonlinear optics in attempt to explain artificial heterogenic media. Such media exhibit new properties, due to 
the resonance type of interaction of the media and light are observed in photonic crystals, random lasers, etc (for a review, see [44]). Some of them can be modeled by the so-called $\mathcal{P}$ - and $\mathcal{P} \mathcal{T}$-symmetric (parity-time) symmetric systems [5, 6, 7, 31, 32, 1, 3, 4, 21, 40].

The initial interest in such systems was motivated by quantum mechanics [6, 31]. In [6] it was shown that quantum systems with a non-hermitian Hamiltonian admit states with real eigenvalues, i.e. the hermiticity of the Hamiltonian is not a necessary condition to have real spectrum. Using such Hamiltonians one can build up new quantum mechanics [6, 7, 31, 32]. Starting point is the fact that in the case of a nonHermitian Hamiltonian with real spectrum, the modulus of the wave function for the eigenstates is time-independent even in the case of complex potentials. All this naturally lead to the development of a special class of non-local versions of the NLS equation and its multicomponent versions [41, 3, 21]. The nonlocality introduced is due to the reductions.

The aim of the present paper is to analyze a special type of MNLS equations of KS type and to show that they preserve integrability also when nonlocal reductions are applied. For $r=3$ and $r=5$ and with the standard (local) reductions they are characterized by the Gross-Pitaevsky energy functionals (see equations (6), (7) below) and correspond to integrable MNLS models related to symmetric spaces [9] of BD.I-type $\simeq \mathrm{SO}(2 \mathrm{r}+1) / \mathrm{SO}(2) \times \mathrm{SO}(2 \mathrm{r}-1)$. Our expose will treat in parallel both reductions.

In Section 2 we give preliminaries about the BEC in one dimension. We also formulate the Lax representations for the KS-type equations for any $r$ Section 3 deals with the direct and inverse scattering problem for the Lax operators. More specifically, we outline the construction of the fundamental analytic solutions (FAS) of $L$ which allows us to reduce the inverse scattering problem (ISP) for $L$ to a Riemann-Hilbert problem (RHP). Such approach allows one to use the ZakharovShabat dressing method for calculating the soliton solutions of the KS equations. All these considerations are valid for Lax operators of generic form, i.e. without any reductions imposed. In Section 4 we formulate the expansions of $q(x, t)$ and its variation $\delta q(x, t)$ over the squared solutions of $L$ for the simplest nontrivial case when $L$ has no discrete eigenvalues. We will see below, that these expansions are compatible with both the local and non-local $\mathbb{Z}_{2}$-reductions. Their expansions coefficients are provided by the minimal sets of scattering data and their variations. They allow one to generalize the idea of [2] also to the multicomponent KS-type equations with both local and nonlocal reductions. Thus we demonstrate that in all these case the ISM is a generalized Fourier transform. In Section 5 we outline the fundamental properties of these NLEE of KS type. In Section 6 we recall Mikhailov's reduction group which can naturally be applied also to nonlocal reductions. We derive the constraints on the scattering data imposed by each of these reductions. 


\section{Preliminaries}

\subsection{The BEC in one dimension}

The main tool for investigating BEC is the Gross-Pitaevski (GP) equation and the GP functional. In the one-dimensional approximation the GP equation in 1D $x$-space becomes:

$$
i \frac{\partial \Phi}{\partial t}=\frac{\delta E_{\mathrm{GP}}[\Phi]}{\delta \Phi^{*}}
$$

where for $F=1$ the GP energy functional is given by:

$$
\begin{aligned}
E_{\mathrm{GP}} & =\int d x\left\{\frac{\hbar^{2}}{2 m}\left|\partial_{x} \Phi\right|^{2}+\bar{c}\left[\left|\Phi_{1}\right|^{4}+\left|\Phi_{-1}\right|^{4}+2\left|\Phi_{0}\right|^{2}\left(\left|\Phi_{1}\right|^{2}+\left|\Phi_{-1}\right|^{2}\right)\right]\right. \\
& \left.+\left(\bar{c}_{0}-\bar{c}_{2}\right)\left|\Phi_{1}\right|^{2}\left|\Phi_{-1}\right|^{2}+\frac{\bar{c}_{0}}{2}\left|\Phi_{0}\right|^{4}+\bar{c}_{2}\left(\Phi_{1}^{*} \Phi_{-1}^{*} \Phi_{0}^{2}+\Phi_{0}^{* 2} \Phi_{1} \Phi_{-1}\right)\right\}
\end{aligned}
$$

For $F=2$ the energy functional is defined by [34, 39, 37]

$$
E_{\mathrm{GP}}[\Phi]=\int_{-\infty}^{\infty} d x\left(\frac{\hbar^{2}}{2 m}\left|\partial_{x} \Phi\right|^{2}+\frac{\varepsilon c_{0}}{2} n^{2}+\frac{c_{2}}{2} \mathbf{f}^{2}+\frac{\varepsilon c_{4}}{2}|\Theta(x, t)|^{2}\right)
$$

where $\varepsilon= \pm 1$. The number density $n$ and the singlet-pair amplitude $\Theta$ are defined in [39, 37]

These two sets of vector NLS eqs. can be viewed as members of another class of MNLS eqs. related to the BD.I type of symmetric spaces. They can be written as [26, 18, 14]:

$$
i \mathbf{q}_{t}+\mathbf{q}_{x x}+2\left(\mathbf{q}, \mathbf{q}^{*}\right) \mathbf{q}-\left(\mathbf{q}, s_{0} \mathbf{q}\right) s_{0} \mathbf{q}^{*}=0,
$$

where $\mathbf{q}$ is $2 r-1$-component vector and the constant matrix $s_{0}$ has nonvanishing elements \pm 1 only on the second diagonal, see eq. (10) below.

\subsection{Lax Representation for BD.I-type MNLS equations}

The symmetric spaces of the series BD.I are isomorphic to $S O(2 r+1) /(S O(2) \otimes$ $S O(2 r-1)$, see [23]. The local coordinates on them are provided by the co-adjoint orbits of the algebras $s o(2 r+1)$ passing through $J=\operatorname{diag}(1,0, \ldots, 0,-1)$. These local coordinates are provided by the matrices $q(x, t)=\sum_{\alpha \in \Delta_{1}^{+}}\left(q_{\alpha} E_{\alpha}+p_{\alpha} E_{-\alpha}\right)$ where the set of roots $\Delta_{1}^{+}=\left\{e_{1}-e_{2}, \ldots, e_{1}-e_{r}, e_{r}, e_{1}+e_{r}, \ldots, e_{1}+e_{2}\right\}$. For the typical representation we have the matrix form: 


$$
q(x, t)=\left(\begin{array}{ccc}
0 & \mathbf{q}^{T} & 0 \\
\mathbf{p} & 0 & s_{0} \mathbf{q} \\
0 & \mathbf{p}^{T} s_{0} & 0
\end{array}\right), \quad J=\operatorname{diag}(1,0, \ldots 0,-1) .
$$

The $2 r-1$-component vectors $\mathbf{q}=\left(q_{2}, \ldots, q_{2 r}\right)^{T}$ are formed by the coefficients $q_{\alpha}$ as follows: $q_{k} \equiv q_{e_{1}-e_{k}}, q_{r+1} \equiv q_{e_{1}}$ and $q_{2 r+1-k} \equiv q_{e_{1}+e_{k}}, k=2, \ldots, r$; the vector $\mathbf{p}=\left(p_{2}, \ldots, p_{n}\right)^{T}$ is formed analogously. The matrix $s_{0}=S_{0}^{(n)}$ enters in the definition of $s o(n)$, i.e. $X \in s o(n)$, if $X+S_{0}^{(n)} X^{T} S_{0}^{(n)}=0$, and for $n=2 r+1$ :

$$
S_{0}^{(n)}=\sum_{s=1}^{n}(-1)^{s+1} E_{s, n+1-s}^{(n)},
$$

With this definition of orthogonality the Cartan subalgebra generators are represented by diagonal matrices. By $E_{s p}^{(n)}$ above we mean $n \times n$ matrix whose matrix elements are $\left(E_{s p}^{(n)}\right)_{i j}=\delta_{s i} \delta_{p j}$.

The MNLS equations allow Lax representation $[L, M]=0$ as follows

$$
\begin{aligned}
L \psi(x, t, \lambda) & \equiv i \partial_{x} \psi+(q(x, t)-\lambda J) \psi(x, t, \lambda)=0 . \\
M \psi(x, t, \lambda) & \equiv i \partial_{t} \psi+\left(V_{0}(x, t)+\lambda V_{1}(x, t)-\lambda^{2} J\right) \psi(x, t, \lambda)=0, \\
V_{1}(x, t) & =q(x, t), \quad V_{0}(x, t)=i \operatorname{ad}_{J}^{-1} \frac{d q}{d x}+\frac{1}{2}\left[\operatorname{ad}_{J}^{-1} q, q(x, t)\right] .
\end{aligned}
$$

In terms of these notations the generic MNLS type equations connected to BD.I. acquire the form

$$
\begin{aligned}
& i \mathbf{q}_{t}+\mathbf{q}_{x x}+2(\mathbf{q}, \mathbf{p}) \mathbf{q}-\left(\mathbf{q}, s_{0} \mathbf{q}\right) s_{0} \mathbf{p}=0, \\
& i \mathbf{p}_{t}-\mathbf{p}_{x x}-2(\mathbf{q}, \mathbf{p}) \mathbf{p}+\left(\mathbf{p}, s_{0} \mathbf{p}\right) s_{0} \mathbf{q}=0,
\end{aligned}
$$

This equation allows two types of reductions. The first one - the typical reduction $\mathbf{p}(x, t)=\mathbf{q}^{*}(x, t)$ is well studied by now, see [27, 9, 22]. The corresponding Hamiltonian for the equations (14) is given by

$$
H_{\mathrm{MNLS}}=\int_{-\infty}^{\infty} d x\left(\left(\partial_{x} \mathbf{q}, \partial_{x} \mathbf{q}^{*}\right)-\left(\mathbf{q}, \mathbf{q}^{*}\right)^{2}+\left(\mathbf{q}, s_{0} \mathbf{q}\right)\left(\mathbf{q}^{*}, s_{0} \mathbf{q}^{*}\right)\right),
$$

For $r=2$ we introduce the variables $\Phi_{1}=q_{2}, \Phi_{0}=q_{3} / \sqrt{2}, \Phi_{-1}=q_{4}$; for $r=3$ we set $\Phi_{2}=q_{2}, \Phi_{1}=q_{3}, \Phi_{0}=q_{4}, \Phi_{-1}=q_{5}$ and $\Phi_{-2}=q_{6}$. This reproduces the action functionals $E_{G P}$ for $F=1$ and $F=2$.

The second reduction is a non-local one $\mathbf{p}(x, t)=-\mathbf{q}^{*}(-x, t)$ and is the main topic of the present paper. As a result we obtain the nonlocal NLS model of BD.Itype:

$$
i \mathbf{q}_{t}+\mathbf{q}_{x x}-2\left(\mathbf{q}(x, t), \mathbf{q}^{*}(-x, t)\right) \mathbf{q}(x, t)+\left(\mathbf{q}(x, t), s_{0} \mathbf{q}(x, t)\right) s_{0} \mathbf{q}^{*}(-x, t)=0 .
$$




\section{The Direct and the Inverse scattering problem.}

Here we will outline the solution of the direct scattering problem and the construction of the fundamental analytic solutions (FAS) [35]. The construction goes true for both choices of involutions: local and nonlocal. Following [43] we reduce it to a RHP.

\subsection{The Direct scattering problem.}

Solving the direct scattering problem for $L$ uses the Jost solutions which are defined by, see [13] and the references therein

$$
\lim _{x \rightarrow-\infty} \phi(x, t, \lambda) e^{i \lambda J x}=\mathbb{1}, \quad \lim _{x \rightarrow \infty} \psi(x, t, \lambda) e^{i \lambda J x}=\mathbb{1}
$$

and the scattering matrix $T(\lambda, t) \equiv \psi^{-1} \phi(x, t, \lambda)$. The choice of $J$ and the fact that the Jost solutions and $T(\lambda, t)$ take values in the group $S O(2 r+1)$ means that we can use the following block-matrix structure of $T(\lambda, t)$

$$
T(\lambda, t)=\left(\begin{array}{ccc}
m_{1}^{+} & -\mathbf{B}^{-T} & c_{1}^{-} \\
\mathbf{b}^{+} & \mathbf{T}_{22} & -s_{0} \mathbf{b}^{-} \\
c_{1}^{+} & \mathbf{B}^{+T} s_{0} & m_{1}^{-}
\end{array}\right), \quad \hat{T}(\lambda, t)=\left(\begin{array}{ccc}
m_{1}^{-} & \mathbf{b}^{-T} & c_{1}^{-} \\
-\mathbf{B}^{+} & s_{0} \mathbf{T}_{22} s_{0} & s_{0} \mathbf{B}^{-} \\
c_{1}^{+} & -\mathbf{b}^{+T} s_{0} & m_{1}^{+}
\end{array}\right),
$$

where $\mathbf{b}^{ \pm}(\lambda, t)$ and $\mathbf{B}^{ \pm}(\lambda, t)$ are $2 r-1$-component vectors, $\mathbf{T}_{22}(\lambda)$ and $m^{ \pm}(\lambda)$ are $2 r-1 \times 2 r-1$ block matrices, and $m_{1}^{ \pm}(\lambda), c_{1}^{ \pm}(\lambda)$ are scalars. The matrix elements of $T(\lambda, t)$ satisfy a number of relations which ensure that $T(\lambda)$ belongs to $S O(2 r+$ 1) and that $T(\lambda) \hat{T}(\lambda)=\mathbb{1}$. Some of them take the form:

$$
\begin{aligned}
& m_{1}^{+} m_{1}^{-}+\mathbf{B}^{-T} \mathbf{B}^{+}+c_{1}^{+} c_{1}^{-}=1, \quad \mathbf{b}^{+} \mathbf{b}^{-T}+T_{22} s_{0} T_{22}^{T} s_{0}+s_{0} \mathbf{b}^{-} \mathbf{b}^{+T} s_{0}=\mathbb{1}, \\
& m_{1}^{+} m_{1}^{-}+\mathbf{b}^{+T} \mathbf{b}^{-}+c_{1}^{+} c_{1}^{-}=1, \quad \mathbf{B}^{+} \mathbf{B}^{-T}+s_{0} T_{22}^{T} s_{0} T_{22}+s_{0} \mathbf{B}^{-} \mathbf{B}^{+T} s_{0}=\mathbb{1} .
\end{aligned}
$$

\subsection{The fundamental analytic solutions}

It is well known that the Jost solutions satisfy a system of Volterra-type integral equations. Indeed, if we introduce

$$
Y_{+}(x, t, \lambda)=\psi(x, t, \lambda) e^{i J \lambda x}, \quad Y_{-}(x, t, \lambda)=\phi(x, t, \lambda) e^{i J \lambda x}
$$

then $Y_{ \pm}(x, t, \lambda)$ must satisfy:

$$
Y_{ \pm ; j k}(x, t, \lambda)=\delta_{j k}+i \int_{ \pm \infty}^{x} d y e^{-i \lambda\left(a_{j}-a_{k}\right)(x-y)}\left([J, Q(y, t)] Y_{ \pm}(x, t, \lambda)\right)_{j k} .
$$


Here we have used the notation $J=\operatorname{diag}\left(a_{1}, a_{2}, \ldots, a_{2 r}, a_{2 r+1}\right)$; i.e. $a_{1}=1, a_{2}=$ $a_{3}=\cdots=a_{2 r}=0, a_{2 r+1}=-1$, (see eq. (9)).

The Volterra equations (20) always have solution for real $\lambda$. Analytic extension for $\lambda \in \mathbb{C}_{+}$(resp. for $\lambda \in \mathbb{C}_{-}$) is possible only for the first column of $Y_{-}(x, t, \lambda)$ and for the last column of $Y_{+}(x, t, \lambda)$ (resp. for the last column of $Y_{-}(x, t, \lambda)$ and for the first column of $Y_{+}(x, t, \lambda)$. Following Shabat's method [35] we consider two sets of integral equations:

$$
\begin{aligned}
& \xi_{j k}^{+}(x, t, \lambda)=\delta_{j k}+i \int_{\varepsilon_{j k}^{\infty}}^{x} d y e^{-i \lambda\left(a_{j}-a_{k}\right)(x-y)}\left([J, Q(y, t)] \xi^{+}(y, t, \lambda)\right)_{j k} . \\
& \xi_{j k}^{-}(x, t, \lambda)=\delta_{j k}+i \int_{-\eta_{j k} \infty}^{x} d y e^{-i \lambda\left(a_{j}-a_{k}\right)(x-y)}\left([J, Q(y, t)] \xi^{-}(y, t, \lambda)\right)_{j k},
\end{aligned}
$$

where

$$
\varepsilon_{j k}=\left\{\begin{array}{ll}
1 & \text { for } \quad j \prec k, \\
-1 & \text { for } j \succeq k,
\end{array}, \quad \eta_{j k}=\left\{\begin{array}{ll}
-1 & \text { for } j \preceq k, \\
1 & \text { for } j \succ k,
\end{array} .\right.\right.
$$

Here we used the notation

$$
\begin{aligned}
& j \prec k \quad \text { iff } \quad a_{j}>a_{k} ; \quad j \preceq k \quad \text { iff } \quad a_{j} \geq a_{k} ; \\
& j \succ k \quad \text { iff } \quad a_{j}<a_{k} ; \quad j \succeq k \quad \text { iff } \quad a_{j} \leq a_{k} \text {. }
\end{aligned}
$$

Then one can prove that the equations (22) (resp. (23) possess solutions $\xi^{+}(x, t, \lambda)$ (resp. $\xi^{-}(x, t, \lambda)$ ) which allow analytic extension for $\lambda \in \mathbb{C}_{+}$(resp. for $\lambda \in \mathbb{C}_{-}$). The solutions $\xi^{ \pm}(x, t, \lambda)$ can be viewed also as solutions to a RHP

$$
\xi^{+}(x, t, \lambda)=\xi^{-}(x, t, \lambda) G(x, t, \lambda), \quad G(x, t, \lambda)=e^{i \lambda J x} G_{0}(t, \lambda) e^{-i \lambda J x}
$$

with canonical normalization, i,e, $\lim _{\lambda \rightarrow \infty} \xi^{ \pm}(x, t, \lambda)=\mathbb{1}$.

If we denote by $\chi^{ \pm}(x, t, \lambda)=\xi^{ \pm}(x, t, \lambda) e^{-i \lambda J x}$ then $\chi^{ \pm}(x, t, \lambda)$ will be the FAS of $L[35,42,12]$. Below we will use two equivalents sets of FAS:

$$
\begin{array}{ll}
\chi^{ \pm}(x, t, \lambda)=\psi(x, t, \lambda) T_{J}^{\mp}(t, \lambda) D_{J}^{ \pm}(\lambda), & \chi^{ \pm}(x, t, \lambda)=\phi(x, t, \lambda) S_{J}^{ \pm}(t, \lambda), \\
\tilde{\chi}^{ \pm}(x, t, \lambda)=\phi(x, t, \lambda) S_{J}^{ \pm}(t, \lambda) \hat{D}_{J}^{ \pm}(\lambda), & \tilde{\chi}^{ \pm}(x, t, \lambda)=\psi(x, t, \lambda) T_{J}^{\mp}(t, \lambda),
\end{array}
$$

where $S_{J}^{ \pm}, T_{J}^{ \pm}$and $D_{J}^{ \pm}$are generalized Gauss factors of the scattering matrix, see [42, 10, 11, 12, 19]:

$$
T(\lambda, t)=T_{J}^{-} D_{J}^{+} \hat{S}_{J}^{+}=T_{J}^{+} D_{J}^{-} \hat{S}_{J}^{-}
$$

where 


$$
\begin{array}{rlrl}
T_{J}^{-}(\lambda, t) & =\left(\begin{array}{ccc}
1 & 0 & 0 \\
\rho^{+} & \mathbb{1} & 0 \\
\tilde{c}_{1}^{-} & \rho^{+T} s_{0} & 1
\end{array}\right), & T_{J}^{+}(\lambda, t) & =\left(\begin{array}{ccc}
1 & -\rho^{-, T} & \tilde{\tilde{c}}_{1}^{+} \\
0 & \mathbb{1} & -s_{0} \rho^{-} \\
0 & 0 & 1
\end{array}\right), \\
S_{J}^{+}(\lambda, t) & =\left(\begin{array}{ccc}
1 & \tau^{+T} & \tilde{c}_{1}^{+} \\
0 & \mathbb{1} & s_{0} \tau^{+} \\
0 & 0 & 1
\end{array}\right), & S_{J}^{-}(\lambda, t) & =\left(\begin{array}{ccc}
1 & 0 & 0 \\
-\tau^{-} & \mathbb{1} & 0 \\
\tilde{c}_{1}^{-} & -\tau^{-T} s_{0} & 1
\end{array}\right), \\
D_{J}^{+}(\lambda)=\left(\begin{array}{ccc}
m_{1}^{+} & 0 & 0 \\
0 & \mathbf{m}_{2}^{+} & 0 \\
0 & 0 & 1 / m_{1}^{+}
\end{array}\right), & D_{J}^{-}(\lambda)=\left(\begin{array}{ccc}
1 / m_{1}^{-} & 0 & 0 \\
0 & \mathbf{m}_{2}^{-} & 0 \\
0 & 0 & m_{1}^{-}
\end{array}\right),
\end{array}
$$

We have made use of the following notations above:

$$
\begin{aligned}
& \rho^{ \pm}=\frac{\mathbf{b}^{ \pm}}{m_{1}^{ \pm}}, \quad \quad \tau^{ \pm}=\frac{\mathbf{B}^{\mp}}{m_{1}^{ \pm}}, \quad \tilde{c}_{1}^{+}=\frac{1}{2}\left(\tau^{+T} s_{0} \tau^{+}\right), \\
& \tilde{c}_{1}^{-}=\frac{1}{2}\left(\rho^{+T} s_{0} \rho^{+}\right), \quad \tilde{c}_{1}^{+}=\frac{1}{2}\left(\rho^{-T} s_{0} \rho^{-}\right), \quad \tilde{c}_{1}^{-}=\frac{1}{2}\left(\tau^{-T} s_{0} \tau^{-}\right), \\
& c_{1}^{+}=\frac{\left(\mathbf{b}^{+T} s_{0} \mathbf{b}^{+}\right)}{2 m_{1}^{+}}, \quad c_{1}^{-}=\frac{\left(\mathbf{B}^{-T} s_{0} \mathbf{B}^{-}\right)}{2 m_{1}^{+}} \text {. }
\end{aligned}
$$

\subsection{The Inverse scattering problem (ISP).}

An important tool for reducing the ISP to a Riemann-Hilbert problem (RHP) are the fundamental analytic solution (FAS) $\chi^{ \pm}(x, t, \lambda)$ and $\tilde{\chi}^{ \pm}(x, t, \lambda)$.

The Lax representation (11), (12) ensures that if $q(x, t)$ evolves according to (14) then the scattering matrix and its elements satisfy the following linear evolution equations

$$
i \frac{d \rho^{ \pm}}{d t} \pm \lambda^{2} \rho^{+}(t, \lambda)=0, \quad i \frac{d \tau^{ \pm}}{d t} \mp \lambda^{2} \tau^{ \pm}(t, \lambda)=0, \quad i \frac{d D^{ \pm}}{d t}=0,
$$

so the block-diagonal matrices $D^{ \pm}(\lambda)$ can be considered as generating functionals of the integrals of motion. The fact that all $(2 r-1)^{2}$ matrix elements of $m_{2}^{ \pm}(\lambda)$ for $\lambda \in \mathbb{C}_{ \pm}$generate integrals of motion reflect the superintegrability of the model and are due to the degeneracy of the dispersion law of 14). We remind that $D_{J}^{ \pm}(\lambda)$ allow analytic extension for $\lambda \in \mathbb{C}_{ \pm}$and that their zeroes and poles determine the discrete eigenvalues of $L$.

Given the solutions $\chi^{ \pm}(x, t, \lambda)$ one recovers $q(x, t)$ via the formula

$$
q(x, t)=\lim _{\lambda \rightarrow \infty} \lambda\left(J-\chi^{ \pm} J \widehat{\chi}^{ \pm}(x, t, \lambda)\right) .
$$

The main goal of the dressing method [42, 10, 19, 24, 22] is, starting from a known solutions $\chi_{0}^{ \pm}(x, t, \lambda)$ of $L_{0}(\lambda)$ with potential $q_{(0)}(x, t)$ to construct new singular solutions $\chi_{1}^{ \pm}(x, t, \lambda)$ of $L$ with a potential $q_{(1)}(x, t)$ with two (or more) addi- 
tional singularities located at prescribed positions $\lambda_{1}^{ \pm}$. It is related to the regular one by a dressing factor $u(x, t, \lambda)$, for details see [13, 20, 24].

\section{The Generalized Fourier Transforms for non-regular $J$}

The generalized Fourier transforms (GFT) for the NLEE are based on the completeness relation for the 'squared solutions' of $L$. These completeness relations for the case of generic $J$ have been proved in [10], see also [11, 21]. In our case $J$ is highly degenerate: $2 r-1$ of its eigenvalues are vanishing. This fact substantially changes the two important steps in the construction:

i) split the algebra $\mathfrak{g} \simeq s o(2 r+1)$ into two subspaces: $\mathfrak{g}=\mathcal{O}_{J} \oplus \mathcal{O}_{J}^{\perp}$. Here $\mathcal{O}_{J}$ is the image of the operator $\operatorname{ad}_{J}$ and provides the co-adjoint orbit in $\mathfrak{g}$ passing through $J$. In our case $\mathcal{O}_{J} \equiv \operatorname{span}\left\{E_{\alpha}, E_{-\alpha}, \alpha \in \delta_{1}^{+}\right\}$. $\mathcal{O}_{J}^{\perp}$ is the complementary space orthogonal to $\mathcal{O}_{J}$ with respect to the Killing form. In what follows we will introduce the operator $\pi_{J}=\operatorname{ad}_{J}^{-1} \operatorname{ad}_{J}$ which projects any element of $\mathfrak{g}$ onto $\mathcal{O}_{J}$;

ii) split each of the 'squared solutions' $e_{\alpha}^{ \pm}(x, \lambda)=\chi^{ \pm}(x, \lambda) E_{\alpha} \hat{\chi}^{ \pm}(x, \lambda)$ and $\tilde{e}_{\alpha}^{ \pm}(x, \lambda)=\tilde{\chi}^{ \pm}(x, \lambda) E_{\alpha} \hat{\tilde{\chi}}^{ \pm}(x, \lambda)$ into two parts:

$$
e_{\alpha}^{ \pm}(x, \lambda)=\mathbf{e}_{\alpha}^{ \pm}(x, \lambda)+e_{\alpha}^{ \pm, \perp}(x, \lambda), \quad \tilde{e}_{\alpha}^{ \pm}(x, \lambda)=\tilde{\mathbf{e}}_{\alpha}^{ \pm}(x, \lambda)+\tilde{e}_{\alpha}^{ \pm, \perp}(x, \lambda),
$$

where $\mathbf{e}_{\alpha}^{ \pm}(x, \lambda), \tilde{\mathbf{e}}_{\alpha}^{ \pm}(x, \lambda)$ belong to $\mathcal{O}_{J}, \mathbf{e}_{\alpha}^{ \pm, \perp}(x, \lambda)$ and $\tilde{\mathbf{e}}_{\alpha}^{ \pm, \perp}(x, \lambda)$ belong to $\mathcal{O}_{J}^{\perp}$.

We can view $q(x, t) \in \mathcal{O}_{J}$ as a generic element of the co-adjoint orbit. The rest of the idea for the GFT is based on the analyticity properties of the 'squared solutions' and on the completeness relation of $\mathbf{e}_{\alpha}^{ \pm}(x, \lambda)$ and $\tilde{\mathbf{e}}_{\alpha}^{ \pm}(x, \lambda), \alpha \in \delta_{1}^{+} \cup\left(-\delta_{1}^{+}\right)$on $\mathcal{O}_{J}$. and is a natural generalization of the proof for generic $J[10,11,13]$. Skipping the details we formulate the expansions for $q(x)$ and ad ${ }_{J}^{-1} \delta q(x)$. Of course, for the sake of brevity we treat the case when the Lax operator $L$ has no discrete eigenvalues.

$$
\begin{aligned}
q(x) & =-\frac{i}{\pi} \int_{-\infty}^{\infty} d \lambda \sum_{\alpha \in \delta_{1}^{+}}\left(\tau_{\alpha}^{+}(\lambda) \mathbf{e}_{\alpha}^{+}(x, \lambda)-\tau_{\alpha}^{-}(\lambda) \mathbf{e}_{-\alpha}^{-}(x, \lambda)\right) \\
& =\frac{i}{\pi} \int_{-\infty}^{\infty} d \lambda \sum_{\alpha \in \delta_{1}^{+}}\left(\rho_{\alpha}^{+}(\lambda) \tilde{\mathbf{e}}_{-\alpha}^{+}(x, \lambda)-\rho_{\alpha}^{-}(\lambda) \tilde{\mathbf{e}}_{\alpha}^{-}(x, \lambda)\right) .
\end{aligned}
$$

Lemma 1. Let the potential $q(x, t)$ be such that the Lax operator L has no discrete eigenvalues. Then as minimal set of scattering data which determines uniquely the scattering matrix $T(\lambda, t)$ and the corresponding potential $q(x, t)$ one can consider either one of the sets $\mathfrak{T}_{i}, i=1,2$

$$
\mathfrak{T}_{1} \equiv\left\{\rho_{\alpha}^{+}(\lambda, t), \rho_{\alpha}^{-}(\lambda, t), \quad \alpha \in \delta_{1}^{+}\right\}, \quad \mathfrak{T}_{2} \equiv\left\{\tau_{\alpha}^{+}(\lambda, t), \tau_{\alpha}^{-}(\lambda, t), \quad \alpha \in \delta_{1}^{+}\right\},
$$

for $\lambda \in \mathbb{R}$. In other words, the minimal sets of scattering data consist of the expansion coefficients of $q(x)$ over the 'squared solutions'.

Similar expansions hold true also for the variation of $q(x)$ [10, 11, 12]: 


$$
\begin{aligned}
\operatorname{ad}_{J}^{-1} \delta q(x) & =\frac{i}{\pi} \int_{-\infty}^{\infty} d \lambda \sum_{\alpha \in \Delta_{1}^{+}}\left(\delta \tau_{\alpha}^{+}(\lambda) \mathbf{e}_{\alpha}^{+}(x, \lambda)+\delta \tau_{\alpha}^{-}(\lambda) \mathbf{e}_{-\alpha}^{-}(x, \lambda)\right) \\
& =\frac{i}{\pi} \int_{-\infty}^{\infty} d \lambda \sum_{\alpha \in \Delta_{1}^{+}}\left(\delta \rho_{\alpha}^{+}(\lambda) \tilde{\mathbf{e}}_{-\alpha}^{+}(x, \lambda)+\delta \rho_{\alpha}^{-}(\lambda) \tilde{\mathbf{e}}_{-\alpha}^{-}(x, \lambda)\right)
\end{aligned}
$$

If we consider the special type of variations: $\delta q(x) \simeq \frac{\partial q}{\partial t} \delta t+\mathcal{O}\left((\delta t)^{2}\right)$, then the expansions (35) go into

$$
\begin{aligned}
\operatorname{ad}_{J}^{-1} \frac{\partial q}{\partial t} & =\frac{i}{\pi} \int_{-\infty}^{\infty} d \lambda \sum_{\alpha \in \Delta_{1}^{+}}\left(\frac{\partial \tau_{\alpha}^{+}}{\partial t} \mathbf{e}_{\alpha}^{+}(x, \lambda)+\frac{\partial \tau_{\alpha}^{-}}{\partial t} \mathbf{e}_{-\alpha}^{-}(x, \lambda)\right) \\
& =\frac{i}{\pi} \int_{-\infty}^{\infty} d \lambda \sum_{\alpha \in \Delta_{1}^{+}}\left(\frac{\partial \rho_{\alpha}^{+}}{\partial t} \tilde{\mathbf{e}}_{-\alpha}^{+}(x, \lambda)+\frac{\partial \rho_{\alpha}^{-}}{\partial t} \tilde{\mathbf{e}}_{-\alpha}^{-}(x, \lambda)\right) .
\end{aligned}
$$

To complete the analogy between the standard Fourier transform and the expansions over the 'squared solutions' we need the generating operators $\Lambda_{ \pm}$:

$$
\Lambda_{ \pm} X(x) \equiv \operatorname{ad}_{J}^{-1}\left(i \frac{d X}{d x}+i\left[q(x), \int_{ \pm \infty}^{x} d y[q(y), X(y)]\right]\right) .
$$

for which the 'squared solutions' are eigenfunctions:

$$
\left(\Lambda_{+}-\lambda\right) \tilde{\mathbf{e}}_{\mp \alpha}^{ \pm}(x, \lambda)=0, \quad\left(\Lambda_{-}-\lambda\right) \mathbf{e}_{ \pm \alpha}^{ \pm}(x, \lambda)=0, \quad \alpha \in \delta_{1}^{+} .
$$

\section{Fundamental properties of the MNLS equations}

The expansions (34), (35) and the explicit form of $\Lambda_{ \pm}$and eq. (38) are basic for deriving the fundamental properties of all MNLS type equations related to the Lax operator $L$. Each of these NLEE is determined by its dispersion law which we choose to be of the form $F(\lambda)=f(\lambda) J$, where $f(\lambda)$ is polynomial in $\lambda$. The corresponding NLEE becomes:

$$
i \operatorname{ad}_{J}^{-1} q_{t}+f\left(\Lambda_{ \pm}\right) q(x, t)=0 .
$$

Theorem 1. The NLEE (39) are equivalent to: $i$ ) the equations (31) and ii) the following evolution equations for the generalized Gauss factors of $T(\lambda)$ :

$$
i \frac{d S_{J}^{+}}{d t}+\left[F(\lambda), S_{J}^{+}\right]=0, \quad i \frac{d T_{J}^{-}}{d t}+\left[F(\lambda), T_{J}^{-}\right]=0, \quad \frac{d D_{J}^{+}}{d t}=0 .
$$

or, equivalently. to:

$$
i \frac{d \tau^{ \pm}}{d t} \mp f(\lambda) \tau^{ \pm}(t, \lambda)=0, \quad i \frac{d \rho^{ \pm}}{d t} \pm f(\lambda) \rho^{ \pm}(t, \lambda)=0 .
$$


The principal series of integrals is generated by the asymptotic expansion of $\ln m_{1}^{+}(\lambda)=\sum_{k=1}^{\infty} I_{k} \lambda^{-k}$. The first integrals of motion are of the form:

$$
I_{1}=-\frac{i}{2} \int_{-\infty}^{\infty} d x\langle q(x), q(x)\rangle, \quad I_{2}=\frac{1}{2} \int_{-\infty}^{\infty} d x\left\langle q_{x}(x), \operatorname{ad}_{J}^{-1} q(x)\right\rangle,
$$

Now $i I_{1}$ can be interpreted as the density of the particles, $I_{2}$ is the momentum. The third one $I_{3}=i H_{\text {MNLS }}$ provides the Hamiltonian. Indeed, the Hamiltonian equations of motion given by $H_{(0)}=-i I_{3}$ with the Poisson brackets

$$
\left\{q_{k}(y, t), p_{j}(x, t)\right\}=i \delta_{k j} \delta(x-y),
$$

coincide with the MNLS equations (14). The above Poisson brackets are dual to the canonical symplectic form:

$$
\left.\Omega_{0}=i \int_{-\infty}^{\infty} d x \operatorname{tr}(\delta \mathbf{p}(x) \wedge, \delta \mathbf{q}(x))=\frac{1}{2 i}\left[\operatorname{ad}_{J}^{-1} \delta q(x) \wedge \wedge_{,} \operatorname{ad}_{J}^{-1} \delta q(x)\right]\right]
$$

where $\wedge$ means that taking the scalar or matrix product we exchange the usual product of the matrix elements by wedge-product.

The Hamiltonian formulation of eq. (14) with $\Omega_{0}$ and $H_{0}$ is just one member of the hierarchy of Hamiltonian formulations provided by:

$$
\Omega_{k}=\frac{1}{i} \llbracket \operatorname{ad}_{J}^{-1} \delta Q \wedge \Lambda^{k} \operatorname{ad}_{J}^{-1} \delta Q \rrbracket, \quad H_{k}=i^{k+3} I_{k+3} .
$$

where $\Lambda=\frac{1}{2}\left(\Lambda_{+}+\Lambda_{-}\right)$. We can also calculate $\Omega_{k}$ in terms of the scattering data variations. Imposing the reduction $q(x)=q^{\dagger}(x)$ we get:

$$
\begin{aligned}
\Omega_{k} & =\frac{1}{2 \pi i} \int_{-\infty}^{\infty} d \lambda \lambda^{k}\left(\Omega_{0}^{+}(\lambda)-\Omega_{0}^{-}(\lambda)\right) \\
& =\frac{1}{2 \pi} \int_{-\infty}^{\infty} d \lambda \lambda^{k} \operatorname{Im}\left(m_{1}^{+}(\lambda)\left(\hat{m}_{2}^{+} \delta \rho^{+}(\lambda) \wedge_{1} \delta \tau^{+}(\lambda)\right)\right) .
\end{aligned}
$$

This allows one to prove that if we are able to cast $\Omega_{0}$ in canonical form, then all $\Omega_{k}$ will also be cast in canonical form and will be pair-wise equivalent.

\section{The consequences of the involutions}

\subsection{Mikhailov's group of reductions}

The notion of the reduction group for the integrable NLEE was introduced by Mikhailov in the beginning of the 1980'ies [29]. 
The reduction group $G_{R}$ is a finite group which preserves the Lax representation (11), (12). This means that the reduction constraints are automatically compatible with the evolution. Mikhailov proposed that $G_{R}$ must act on the Lax pair with its two realizations simultaneously: i) $G_{R} \subset$ Autg and ii) $G_{R} \subset \operatorname{Conf} \mathbb{C}$, i.e. as conformal mappings of the complex $\lambda$-plane. To each $g_{k} \in G_{R}$ we relate a reduction condition for the Lax pair as follows [29]:

$$
C_{k}\left(L\left(\Gamma_{k}(\lambda)\right)\right)=\eta_{k} L(\lambda), \quad C_{k}\left(M\left(\Gamma_{k}(\lambda)\right)\right)=\eta_{k} M(\lambda),
$$

where $C_{k} \in$ Aut $\mathfrak{g}$ and $\Gamma_{k}(\lambda) \in \operatorname{Conf} \mathbb{C}$ are the images of $g_{k}$ and $\eta_{k}=1$ or -1 depending on the choice of $C_{k}$. Since $G_{R}$ is a finite group then for each $g_{k}$ there exist an integer $N_{k}$ such that $g_{k}^{N_{k}}=\mathbb{1}$. In all the cases below $N_{k}=2$ and the reduction group is isomorphic to $\mathbb{Z}_{2}$.

More specifically the automorphisms $C_{k}, k=1, \ldots, 4$ listed above lead to the following reductions for the matrix-valued functions

$$
U(x, t, \lambda)=[J, Q(x, t)]-\lambda J, \quad V(x, t, \lambda)=V_{0}(x, t)+\lambda V_{1}(x, t)-\lambda^{2} J,
$$

of the Lax representation:

$$
\begin{aligned}
& \text { 1) } \quad C_{1}\left(U^{\dagger}\left(\kappa_{1}(\lambda)\right)\right)=U(\lambda), \quad C_{1}\left(V^{\dagger}\left(\kappa_{1}(\lambda)\right)\right)=V(\lambda) \text {, } \\
& \text { 2) } \quad C_{2}\left(U^{T}\left(\kappa_{2}(\lambda)\right)\right)=-U(\lambda), \quad C_{2}\left(V^{T}\left(\kappa_{2}(\lambda)\right)\right)=-V(\lambda) \text {, } \\
& \text { 3) } \quad C_{3}\left(U^{*}\left(\kappa_{1}(\lambda)\right)\right)=-U(\lambda), \quad C_{3}\left(V^{*}\left(\kappa_{1}(\lambda)\right)\right)=-V(\lambda) \text {, } \\
& \text { 4) } \quad C_{4}\left(U\left(\kappa_{2}(\lambda)\right)\right)=U(\lambda), \quad C_{4}\left(V\left(\kappa_{2}(\lambda)\right)\right)=V(\lambda) \text {, }
\end{aligned}
$$

For the nonlocal involutions we change also $x \rightarrow-x$ and find:

$$
\begin{aligned}
\text { 1) } & C_{1}\left(U^{\dagger}\left(\kappa_{1}(\lambda)\right)\right)=-U(\lambda), & & C_{1}\left(V^{\dagger}\left(\kappa_{1}(\lambda)\right)\right)=V(\lambda), \\
2) & C_{2}\left(U^{T}\left(\kappa_{2}(\lambda)\right)\right)=U(\lambda), & & C_{2}\left(V^{T}\left(\kappa_{2}(\lambda)\right)\right)=-V(\lambda), \\
3) & C_{3}\left(U^{*}\left(\kappa_{1}(\lambda)\right)\right)=U(\lambda), & & C_{3}\left(V^{*}\left(\kappa_{1}(\lambda)\right)\right)=-V(\lambda), \\
4) & C_{4}\left(U\left(\kappa_{2}(\lambda)\right)\right)=-U(\lambda), & & C_{4}\left(V\left(\kappa_{2}(\lambda)\right)\right)=V(\lambda),
\end{aligned}
$$

Both types of involutions impose constraints on the scattering matrix and on its Gauss factors that are listed below.

\subsection{The local involution case}

The involution:

$$
U^{\dagger}\left(x, t, \kappa_{1} \lambda^{*}\right)=U(x, t, \lambda), \quad \Leftrightarrow \quad q(x, t)=q^{\dagger}(x, t), \quad \kappa_{1}=1,
$$

On the Jost solutions we have 


$$
\phi^{\dagger}\left(x, t, \lambda^{*}\right)=\phi^{-1}(x, t, \lambda), \quad \psi^{\dagger}\left(x, t, \lambda^{*}\right)=\psi^{-1}(x, t, \lambda),
$$

so for the scattering matrix we have

$$
T^{\dagger}\left(t, \lambda^{*}\right)=T^{-1}(t, \lambda)
$$

and for the Gauss factors:

$$
S^{-\dagger}\left(\lambda^{*}\right)=\hat{S}^{+}(\lambda), \quad T^{-\dagger}\left(\lambda^{*}\right)=\hat{T}^{-}(\lambda), \quad D^{-\dagger}\left(\lambda^{*}\right)=\hat{D}^{+}(\lambda),
$$

Note that the FAS can be used to define the kernel of the resolvent of $L$ by $R^{ \pm}(x . y . \lambda)=-i \chi^{ \pm}(x, \lambda) \Theta^{ \pm}(x-y) \hat{\chi}^{ \pm}(y, \lambda)$, where the functions $\Theta^{ \pm}(x-y)$ satisfy the equation $\frac{\partial}{\partial x} \Theta^{ \pm}(x-y)=\delta(x-y) \mathbb{1}$ [12, 13]. Next, one can fix up $\Theta^{ \pm}(x-y)$ in such a way that $R^{ \pm}(x, y, \lambda)$ fall off exponentially for $x, y \rightarrow \pm \infty$. So, if $D^{+}(\lambda)$ (or $D^{-}(\lambda)$ ) have a zero or a pole at $\lambda=\lambda_{1}^{+}$(or at $\lambda=\lambda_{1}^{-}$) then $\lambda_{1}^{ \pm}$will be poles of $R^{ \pm}(x, y, \lambda)$ and consequently, discrete eigenvalues of $L$.

If we have local reduction, then

$$
\tau^{+}(\lambda)=-\tau^{-, *}(\lambda), \quad \rho^{+}(\lambda)=-\rho^{-, *}(\lambda),
$$

\subsection{The nonlocal involution case}

Now the involution is:

$$
U^{\dagger}\left(x, t, \lambda^{*}\right)=-U(-x, t,-\lambda), \quad \Leftrightarrow \quad q(x, t)=q^{\dagger}(-x, t) .
$$

On the Jost solutions we have

$$
\phi^{\dagger}\left(x, t, \lambda^{*}\right)=\psi^{-1}(-x, t,-\lambda), \quad \psi^{\dagger}\left(x, t, \lambda^{*}\right)=\phi^{-1}(x, t,-\lambda),
$$

so for the scattering matrix we have

$$
T^{\dagger}\left(t,-\lambda^{*}\right)=T(t, \lambda)
$$

As a consequence for the Gauss factors we get:

$$
T^{-\dagger}\left(-\lambda^{*}\right)=\hat{S}^{+}(\lambda), \quad T^{+\dagger}\left(-\lambda^{*}\right)=\hat{S}^{-}(\lambda), \quad D^{ \pm \dagger}\left(\lambda^{*}\right)=\hat{D}^{ \pm}(-\lambda) .
$$

In analogy with the local reductions, the kernel of the resolvent has poles at the at the points $\lambda_{2}^{ \pm}$at which $D^{ \pm}(\lambda)$ have poles or zeroes. In particular, if $\lambda_{2}^{+}$is an eigenvalue, then $-\lambda_{2}^{+}$is also an eigenvalue. For the reflection coefficients we obtain the constraints:

$$
\tau^{+}(-\lambda)=-\rho^{+, *}(\lambda), \quad \tau^{-}(-\lambda)=-\rho^{-, *}(\lambda),
$$




\section{Conclusion}

We demonstrated that the results concerning the GFT for nonlocal reductions hold true also for the MNLS cases, in particular for the Kulish-Sklyanin type models. The results are natural extensions of the ones in [21] to the multicomponent cases.

\section{References}

1. M. Ablowitz, I. Bakirtas and B. Ilan. Wave collapse in a class of nonlocal nonlinear Schrddinger equation, Physica D 207, 230253, (2005).

2. M.J. Ablowitz, D.J. Kaup, A.C. Newell and H. Segur, The Inverse Scattering TransformFourier Analysis for Nonlinear Problems, Stud. Appl. Math. 53, 249-315 (1974).

3. M. Ablowitz and Z. Musslimani, Integrable Nonlocal Nonlinear Schrödinger Equation, Phys. Rev. Lett., 110 (2013) 064105(5).

4. Mark J. Ablowitz and Ziad H. Musslimani. Inverse scattering transform for the integrable nonlocal nonlinear Schrödinger equation, under review (2015).

5. I.V. Barashenkov, D.A. Zezyulin, V.V. Konotop. Exactly solvable Wadati potentials in the PTsymmetric Gross-Pitaevskii equation arXiv preprint arXiv:1511.06633

6. C. M. Bender and S. Boettcher, Real Spectra in Non-hermitian Hamiltonians Having $\mathcal{P} \mathcal{T}$ Symmetry, Phys. Rev. Lett 80 (1998) 5243-5246;

C. M. Bender, S. Boettcher and P. N. Meisinger, P T-Symmetric quantum Mechanics, J. Math. Phys. 40 (1999) 2201-2229.

7. C. M. Bender, Making Sense of Non-hermitian Hamiltonians, Rep. Progr. Phys. 70 (2007) 947-1018 (E-print:hep-th/0703096).

8. Doktorov, E. V., Wang, J. and Yang, J., "Perturbation theory for bright spinor Bose-Einstein condensate solitons", Phys. Rev. A 77, 043617 (2008).

9. A. P. Fordy and P. P. Kulish, Nonlinear Schrodinger Equations and Simple Lie Algebras, Commun. Math. Phys. 89 (1983) 427-443.

10. Gerdjikov V. S. Generalized Fourier Transforms for the Soliton Equations. Gauge Covariant Formulation. Inverse Problems 2, n. 1 (1986) 51-74.

11. Gerdjikov V. S. Complete Integrability, Gauge Equivalence and Lax Representations of the Inhomogeneous Nonlinear Evolution Equations. Theor. Math. Phys. 92 (1992) 374-386.

12. V. S. Gerdjikov. Algebraic and Analytic Aspects of $N$-wave Type Equations. Contemporary Mathematics 301, 35-68 (2002); nlin.SI/0206014

13. V. S. Gerdjikov. Basic Aspects of Soliton Theory. In: Eds.: I. M. Mladenov, A. C. Hirshfeld. "Geometry, Integrability and Quantization", pp. 78-125; Softex, Sofia 2005.nlin.SI/0604004

14. V. S. Gerdjikov, G. G. Grahovski. Multi-component NLS Models on Symmetric Spaces: Spectral Properties versus Representations Theory. SIGMA 6 (2010), 044, 29 pages; arXiv: 1006.0301 [nlin.SI].

15. V. S. Gerdjikov, G. G. Grahovski, N. A. Kostov. On the multi-component NLS type equations on symmetric spaces and their reductions. Theor. Math. Phys. 144 No. 2 1147-1156 (2005).

16. V.S. Gerdjikov, N.A. Kostov, T.I. Valchev. $N$-Wave Equations with Orthogonal Algebras: $\mathbb{Z}_{2}$ and $\mathbb{Z}_{2} \times \mathbb{Z}_{2}$ Reductions and Soliton Solutions. SIGMA 3, paper 039 (2007); 19 pages. arXiv:nlin.SI/0703002

17. V. S. Gerdjikov, N. A. Kostov, T. I. Valchev. Solutions of multi-component NLS models and Spinor Bose-Einstein condensates. Physica D 238 1306-1310 (2009) arXiv:0802.4398 [nlin.SI].

18. V. S. Gerdjikov, N. A. Kostov and T. I. Valchev. Bose-Einstein condensates with $F=1$ and $F=2$. Reductions and soliton interactions of multi-component NLS models. In (eds: Solomon M. Saltiel; Alexander A. Dreischuh; Ivan P. Christov) Proceedings of SPIE 7501, 7501W (2009). arXiv: 1001.0168 [nlin.SI] 
19. V. S. Gerdjikov, G. G. Grahovski, R. I. Ivanov and N. A. Kostov, $N$-wave interactions related to simple Lie algebras. $\mathbb{Z}_{2}$ - reductions and Soliton Solutions, Inv. Problems 17 (2001) 9991015.

20. V. S. Gerdjikov, N. A. Kostov, T. I. Valchev. Solutions of multi-component NLS models and Spinor Bose-Einstein condensates, Physica D 238 1306-1310 (2009); arXiv:0802.4398 [nlin.SI].

21. V. S. Gerdjikov, A. Saxena. Complete integrability of Nonlocal Nonlinear Schrödinger equation. arXiv:1510.00480 1 [nlin.SI].

22. G. G. Grahovski, V. S. Gerdjikov, N. A. Kostov, V. A. Atanasov, New Integrable Multicomponent NLS type Equations on Symmetric Spaces: $Z_{4}$ and $Z_{6}$ reductions, In "Geometry, Integrability and Quantization VII”, Eds: I. Mladenov and M. De Leon, Softex, Sofia (2006), pp. $154-175$.

23. S. Helgasson, Differential Geometry, Lie Groups and Symmetric Spaces, (Graduate studies in Mathematics, vol.34), AMS, Providence, Rhode Island (2001).

24. R. I. Ivanov, On the dressing method for the generalized Zakharov-Shabat system, Nucl. Phys. B 694, (2004) 509-524.

25. Nistazakis, H. E., Frantzeskakis, D. J., Kevrekidis, P. G., Malomed, B. A. and CarreteroGonzalez R., "Bright-Dark Soliton Complexes in Spinor Bose-Einstein Condensates" Phys. Rev. A 77, 033612 (2008).

26. N. A. Kostov, V. A. Atanasov, V. S. Gerdjikov, G. G. Grahovski. On the soliton solutions of the spinor Bose-Einstein condensate . Proceedings of SPIE 6604, 66041T (2007). Editors: Peter A. Atanasov, Tanja N. Dreischuh, Sanka V. Gateva, Lubomir M. Kovachev.

27. P. P. Kulsh and E. K. Sklyanin. O(n)-invariant nonlinear Schrödinger equation a new completely integrable system. Phys. Lett. A 84A 349-352 (1981).

28. Manakov, S.V.: On the theory of two-dimensional stationary self-focusing of electromagnetic waves. Zh. Eksp. Teor. Fiz. 65, 1392 (1973). (English translation) Sov. Phys. JETP 38, 248 (1974). (in Russian)

29. A. V. Mikhailov, The reduction problem and the inverse scattering problem, Physica D 3 (1981) 73-117.

30. J. Ieda, T. Miyakawa and M. Wadati, Matter-wave solitons in an $F=1$ spinor Bose-Einstein condensate, J. Phys. Soc. Jpn. 73 (2004) 2996.

31. A. Mostafazadeh, Pseudo-hermiticity versus $\mathcal{P} \mathcal{T}$-Symmetry I, II, III, J. Math. Phys. 43 (2002) 205-214 (E-print: math-ph/0107001); 2814-2816 (E-print: math-ph/0110016); 3944-3951 (E-print: math-ph/0203005).

32. A. Mostafazadeh, Pseudo-hermiticity and Generalized $\mathcal{P} \mathcal{T}$ - and $\mathcal{C P} \mathcal{P}$-Symmetries, J. Math. Phys. 44 (2003) 974-989 (E-print: math-ph/0209018); A. Mostafazadeh, Exact $\mathcal{P} \mathcal{T}$-Symmetry Is Equivalent to Hermiticity, J. Phys. A: Math. Gen. 36 (2003) 7081-7091 (E-print: quant-ph/0304080).

33. Nille N. Klausen, John L. Bohn and Chris H. Greene. Nature of spinor Bose-Einstein condensates in rubidium. Phys. Rev. A 64, 053602 (2001).

34. T. Ohmi and K. Machida, Bose-Einstein condensation with internal degrees of freedom in alkali atom gases J. Phys. Soc. Jpn. 67 (1998) 1822.

35. A. B. Shabat. The inverse scattering problem for a system of differential equations. Functional Annal. \& Appl. 9, n.3, 75 (1975) (In Russian);

A. B. Shabat. The inverse scattering problem. Diff. Equations 15, 1824 (1979) (In Russian).

36. M. Uchiyama, J. Ieda and M. Wadati, Dark solitons in $F=1$ spinor Bose-Einstein condensate J. Phys. Soc. Jpn. 75 (2006) 064002.

37. M. Uchiyama, J. Ieda, and M. Wadati, Multicomponent Bright Solitons in F $=2$ Spinor BoseEinstein Condensates, J. Phys. Soc. Japan,76, No. 7, (2007), 74005.

38. Uchino, Shun, Otsuka, Takaharu, Ueda, Masahito. Dynamical symmetry in spinor BoseEinstein condensates arXiv:0710.5210

39. M. Ueda and M. Koashi, Theory of spin-2 Bose-Einstein condensates: Spin correlations, magnetic response, and excitation spectra, Phys. Rev. A 65 (2002) 063602. 
40. T. Valchev. On a nonlocal nonlinear Schrödinger equation, in "Mathematics in Industry", Ed. A. Slavova, Cambridge Scholars Publ., 2014, 36-52, ISBN 978-1-4438-6401-5 (Proc. of 8-th Annual Meeting of the Bulgarian Section of SIAM, 18-19 December, 2013, Sofia, Bulgaria).

41. T. I. Valchev, On Mikhailov's reduction group, Phys. Lett A 379 (2015) 1877-1880.

42. V. E. Zakharov, S. V. Manakov, S. P. Novikov and L. I. Pitaevskii, Theory of Solitons. The Inverse Scattering Method, Plenum Press (Consultant Bureau), N.Y., (1984).

43. V. E. Zakharov, and A. B. Shabat, A scheme for integrating nonlinear evolution equations of mathematical physics by the inverse scattering method. I \& II, Funkts. Anal. Prilozhen., 8 (1974), 43-53; 13 (1979) no. 3, 13-22.

44. A. A. Zyablovsky, A. P. Vinogradov, A. A. Pukhov, A. V. Dorofeenko and A A Lisyansky, $\mathcal{P} \mathcal{T}$-symmetry in optics, Phys.-Uspekhi 57 (2014), no. 11, 1063. 